PROCEEDINGS OF THE

AMERICAN MATHEMATICAL SOCIETY

Volume 128, Number 5, Pages 1357-1360

S 0002-9939(99)05127-8

Article electronically published on October 18, 1999

\title{
FACTORING WEAKLY COMPACT OPERATORS AND THE INHOMOGENEOUS CAUCHY PROBLEM
}

\author{
DIÓMEDES BÁRCENAS
}

(Communicated by David R. Larson)

\begin{abstract}
By using the technique of factoring weakly compact operators through reflexive Banach spaces we prove that a class of ordinary differential equations with Lipschitz continuous perturbations has a strong solution when the problem is governed by a closed linear operator generating a strongly continuous semigroup of compact operators.
\end{abstract}

\section{INTRODUCTION}

Consider a Banach space $X$ and the abstract Cauchy problem

$$
\left\{\begin{array}{l}
\dot{x}(t)=A x+f(t, x), \\
x\left(t_{0}\right)=x_{0} \in D(A)
\end{array}\right.
$$

where $0 \leq t_{0}<T<\infty$ and $A$ generates a strongly continuous semigroup $\left\{T_{t}\right\}_{t \geq 0}$. It is known that the problem (1) does not have to have any solution on $\left[t_{0}, T\right]$ as can be seen by considering a variation of an example given in [4, Chapter $\mathrm{X}$, exercise 5 , section X.5, if $X=c_{0}, f(t, x)=y$ where $y_{n}=\sqrt{\left|x_{n}\right|}$ and $A=0$.

In [6] it is proved that if $f$ is Lipschitz continuous in both variables, then (1) has always a mild solution; but according to Webb [7], this mild solution does not need to be a strong solution.

The strongness of a mild solution of (11) is obtained by Pazy [6], p. 189, according to the following hypothesis:

If $f:[0, T] \times X \rightarrow X$ is Lipschitz continuous in both variables and $X$ is a reflexive Banach space, then a mild solution of (1) is a strong solution.

In this paper we use the factorization scheme announced in the abstract in order to show that the same conclusion holds in non-reflexive Banach spaces when some extra hypotheses are imposed on either the operator $A$ or the perturbation $f$.

Received by the editors December 3, 1997 and, in revised form, June 22, 1998.

1991 Mathematics Subject Classification. Primary 34C10; Secondary 47H20.

Key words and phrases. Semigroup of compact operators, Lipschitz continuous functions, strong solutions.

This research was partially supported by CDCHT of ULA under project C840-97. 


\section{The REsults}

We start with the following definitions:

Definition 2.1. A function $x:\left[t_{0}, T\right] \rightarrow X$ is called a mild solution of (1) if

$$
x(t)=T_{t-t_{0}} x_{0}+\int_{t_{0}}^{t} T_{t-s} f(s, x(s)) d s .
$$

A mild solution $x$ of (11) is called strong solution if $x$ is differentiable almost everywhere with

$$
x^{\prime} \in L_{\left[t_{0}, T ; X\right]}^{1} \quad \text { and } \quad x^{\prime}(t)=A x(t)+f(t, x(t))
$$

for almost every $t$ in $\left[t_{0}, T\right]$.

Lemma 2.1. If $A$ generates a strongly continuous semigroup of weakly compact operators, then, for each $t^{\prime}>0$, the problem

$$
\left\{\begin{array}{l}
\dot{x}(t)=A x(t)+T_{t^{\prime}} f(t), \\
x\left(t_{0}\right)=x_{0} \in D(A)
\end{array}\right.
$$

has a strong solution on $\left[t_{0}, T\right]$ whenever $f:\left[t_{0}, T\right] \rightarrow X$ is Lipschitz continuous.

Proof. Since $T_{t}^{\prime}$ is weakly compact, then by 2] there are a reflexive Banach space $Z$ and bounded linear operators $u, v$ such that

$$
T_{t}^{\prime}=u \circ v, \quad v: X \rightarrow Z, u: Z \rightarrow X .
$$

$v f:\left[t_{0}, T\right] \rightarrow Z$ is then Lipschitz continuous and by the reflexivity of $Z$, $v f$ is differentiable almost everywhere with derivative belonging to $L_{\left[t_{0}, T ; Z\right]}^{1}$. This implies that $T_{t^{\prime}} f=u \circ v f:\left[t_{0}, T\right] \rightarrow X$ is differentiable almost everywhere with derivative belonging to $L_{\left[t_{0}, T ; X\right]}^{1}$, so by $[6]$, Corollary 4.2 .10 , the proof is over.

Theorem 2.1. Suppose that A generates a strongly continuous compact semigroup of bounded linear operators and $f:\left[t_{0}, T\right] \rightarrow X$ is Lipschitz. Then the Cauchy problem

$$
\left\{\begin{array}{l}
\dot{x}(t)=A x(t)+f(t) \\
x\left(t_{0}\right)=x_{0} \in D(A)
\end{array}\right.
$$

has a strong solution on $\left[t_{0}, T\right]$.

Proof. Take a decreasing sequence of positive numbers $t_{n}$ going to 0 . Then, by Lemma 2.1, for each $n \in N$, the Cauchy problem

$$
\left\{\begin{array}{l}
\dot{x}(t)=A x(t)+T_{t_{n}} f(t), \\
x\left(t_{0}\right)=x_{0} \in D(A)
\end{array}\right.
$$

has a strong solution $x_{n}$ given by

$$
\begin{aligned}
x_{n}(t) & =T_{t-t_{0}} x_{0}+\int_{t_{0}}^{t} T_{t-s} T_{t_{n}} f(s) d s \\
& =T_{t-t_{0}} x_{0}+T_{t_{n}}\left(\int_{t_{0}}^{t} T_{t-s} f(s) d s\right) .
\end{aligned}
$$


Now, we notice that, for $t \in\left[t_{0}, T\right]$,

(1) $\lim _{n \rightarrow \infty} x_{n}(t)=T_{t-t_{0}} x_{0}+\int_{t_{0}}^{t} T_{t-s} f(s) d s=x(t)$.

(2) For each $n \in \mathbb{N}, x_{n}^{\prime}(t)$ exists almost everywhere,

$$
x_{n}^{\prime}(t)=A T_{t-t_{0}} x_{0}+T_{t-t_{0}} T_{t_{n}} f\left(t_{0}\right)+\int_{t_{0}}^{t} T_{t-s}\left(T_{t_{n}} f\right)^{\prime}(s) d s
$$

and $x_{n}^{\prime} \in L_{\left[t_{0}, T ; X\right]}$.

Since $f$ is Lipschitz continuous, there is $K>0$ so that

$$
\|f(t)-f(s)\| \leq K\|s-t\| \quad \forall s, t \in\left[t_{0}, T\right] .
$$

Considering that $\left\{T_{t}\right\}_{t \geq 0}$ is a strongly continuous semigroup, we find $M>0$ so that $\left\|T_{t}\right\| \leq M \quad \forall t \in\left[t_{0}, T\right]$. Therefore

$$
\sup _{n, s}\left\|\left(T_{t_{n}} f\right)^{\prime}(s)\right\| \leq K M,
$$

which implies that $\left\{\left(T_{t_{n}} f\right)^{\prime}(s)\right\}_{n=1}^{\infty}$ is uniformly integrable in $L_{\left[t_{0}, T ; X\right]}^{1}$.

Since $\left\{T_{t}\right\}_{t \geq 0}$ is a compact semigroup, by [5] (alternatively [1])

$$
y_{n}(\cdot)=\int_{t_{0}}^{\cdot} T_{-s}\left(T_{t_{n}} f\right)^{\prime}(s) d s
$$

has a subsequence relabeled as $y_{n}$, converging to $g$ in the uniform topology of $C_{\left[t_{o}, T ; X\right]}$, so for almost every $t \in\left[t_{0}, T\right]$,

$$
\lim _{n \rightarrow \infty} x_{n}^{\prime}=\lim _{n \rightarrow \infty} A T_{t-t_{0}} x_{0}+T_{t-t_{0}} f\left(t_{0}\right)+g(t),
$$

which implies that, for almost every $t \in\left[t_{0}, T\right]$,

$$
\lim _{n \rightarrow \infty} x_{n}(t)=\int_{t_{0}}^{t}\left(g(s)+A T_{t-t_{o}} x_{0}+T_{t-t_{0}} f\left(t_{0}\right)\right) d s
$$

and this implies that

$$
x(t)=\int_{t_{0}}^{t}\left(g(s)+A T_{t-t_{0}} x_{0}+T_{t-t_{0}} f\left(t_{0}\right)\right) d s .
$$

Hence,

$$
x^{\prime}(t)=A T_{t-t_{0}} x_{0}+T_{t-t_{0}} f\left(t_{0}\right)+g(t)
$$

almost everywhere. This means that $x$ is differentiable almost everywhere and $x^{\prime} \in L_{\left[t_{0}, T ; X\right]}^{1}$

Under additional hypotheses, the strong compactness of $T_{t}$ can be removed.

Theorem 2.2. Suppose that A generates a strongly continuous semigroup of weakly compact operators and $f:\left[t_{0}, T\right] \rightarrow X$ is Lipschitz continuous and $\left\{t_{n}\right\}_{n=1}^{\infty}$ is a sequence as in foregoing theorem. If there is a compact subset $K$ of $X$ for which the sequence of derivatives $\left(T_{t_{n}} f^{\prime}\right)(s) \in K$ for every $n$ and almost every $s$, then the Cauchy problem

$$
\left\{\begin{array}{l}
\dot{x}(t)=A x(t)+f(t), \\
x\left(t_{0}\right)=x_{0}
\end{array}\right.
$$

has a strong solution on $\left[t_{0}, T\right]$. 
Proof. By Lemma 2.1 and Theorem 6.2 of [1], the sequence $\left\{y_{n}\right\}$ defined by

$$
y_{n}(t)=\int_{t_{0}}^{t} T_{t-s}\left(T_{t_{n}} f\right)^{\prime}(s) d s
$$

is relatively compact in $C_{\left[t_{0}, T ; X\right]}$ and the proof follows as in the above theorem.

Combining the techniques used in the proofs of Theorem 2.1 and Theorem 2.2 together with that of [6] in Theorem 1.6 of Chapter 6 , we obtain:

Theorem 2.3. If A generates a strongly continuous semigroup of compact operators and $f:\left[t_{o}, T\right] \times X \rightarrow X$ is Lipschitz continuous in both variables, then the Cauchy problem

$$
\left\{\begin{array}{l}
\dot{x}(t)=A x(t)+f(t, x), \\
x\left(t_{0}\right)=x_{0} \in D(A)
\end{array}\right.
$$

has a strong solution on $\left[t_{o}, T\right]$.

Theorem 2.4. If A generates a semigroup of weakly compact operators, $f:\left[t_{0}, T\right] \times$ $X \rightarrow X$ is Lipschitz continuous in both variables, $\left\{t_{n}\right\}$ is a sequence of positive numbers going to zero, and $K$ a compact subset of $X$ for which $\left(T_{t_{n}} f\right)^{\prime}(s) \in K$ for each $n \in N$ and almost every $s \in\left[t_{0}, T\right]$, then (6) has a strong solution on $\left[t_{0}, T\right]$.

Remark. An important class of differential equations on which our results find applications are the so-called delay equations, which have the particularity of being the semigroup strongly compact for time greater than or equal to the delaying time, say $t^{\prime}$ (see 3 for a recent reference). We also notice that the diffusion process also generates compact semigroups ([6], p. 234).

\section{REFERENCES}

[1] D. Bárcenas and W. Urbina, Measurable Multifunctions in non separable Banach spaces, SIAM, J. Math. Anal. 28, (1997), 1212-1226. CMP 97:17

[2] W. J. Davis, T. Fiegel, W. B. Johnson and A. Pelczynsky, Factoring weakly compact Operators, J. Functional Analysis, 17, (1974), 311-327. MR 50:8010

[3] O. Diekman, S. A. van Gils, S. M. Verduyn Lunel and H. O. Wather, Delay equations: Functional, complex and nonlinear analysis, Springer Verlag, Berlin, 1995.

[4] J. Dieudonne, Foundations of modern analysis, Academic Press, New York (1967).

[5] H. O. Fatorini, Relaxation in semilinear infinite dimensional control systems, Differential equations, Dynamical systems and control science, Marcel Dekker, New York (1993), 505-522.

[6] A. Pazy, Semigroup of linear operators and applications to partial differential equations, Springer Verlag, Berlin (1983). MR 85g:47061

[7] G. F. Webb, Continuous nonlinear perturbations of linear accretive operators in Banach spaces, J. Funct. Anal., 10, (1972), 191-203. MR 50:14407

Departamento de Mathemáticas, Universidad de los Andes, Mérida 5101, Venezuela

E-mail address: barcenas@ciens.ula.ve 